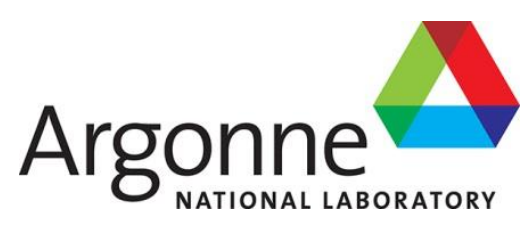

ANL-18/31

\title{
Assessment of Model-Based Schemes for Accelerating Optimization by RAVEN
}

Nuclear-Renewable Hybrid Energy Systems: Analysis of Technical \& Economic Issues

Nuclear Science and Engineering Division 


\title{
About Argonne National Laboratory
}

Argonne is a U.S. Department of Energy laboratory managed by UChicago Argonne, LLC under contract DE-AC02-06CH11357. The Laboratory's main facility is outside Chicago, at 9700 South Cass Avenue, Argonne, Illinois 60439. For information about Argonne and its pioneering science and technology programs, see www.anl.gov.

\section{Document availability}

Online Access: U.S. Department of Energy (DOE) reports produced after 1991 and a growing number of pre-1991 documents are available free at OSTI.GOV

(http://www.osti.gov/), a service of the U.S. Dept. of Energy's Office of Scientific and

Technical Information

\author{
Reports not in digital format may be purchased by the public from the \\ National Technical Information Service (NTIS): \\ U.S. Department of Commerce \\ National Technical Information Service \\ 5301 Shawnee Rd \\ Alexandria, VA 22312 \\ www.ntis.gov \\ Phone: (800) 553-NTIS (6847) or (703) 605-6000 \\ Fax: (703) 605-6900 \\ Email: orders@ntis.gov
}

\section{Reports not in digital format are available to DOE and DOE contractors from the} Office of Scientific and Technical Information (OSTI):

U.S. Department of Energy

Office of Scientific and Technical Information

P.O. Box 62

Oak Ridge, TN 37831-0062

www.osti.gov

Phone: (865) 576-8401

Fax: (865) 576-5728

Email: reports@osti.gov

\section{Disclaimer}

This report was prepared as an account of work sponsored by an agency of the United States Government. Neither the United States Government nor any agency thereof, nor UChicago Argonne, LLC, nor any of their employees or officers, makes any warranty, express or implied, or assumes any legal liability or responsibility for the accuracy, completeness, or usefulness of any information, apparatus, product, or process disclosed, or represents that its use would not infringe privately owned rights. Reference herein to any specific commercial product, process, or service by trade name, trademark, manufacturer, or otherwise, does not necessarily constitute or imply its endorsement, recommendation, or favoring by the United States Government or any agency thereof. The views and opinions of document authors expressed herein do not necessarily state or reflect those of the United States Government or any agency thereof, Argonne National Laboratory, or UChicago Argonne, LLC. 


\section{Assessment of Model-Based Schemes for Accelerating Optimization by RAVEN}

Nuclear-Renewable Hybrid Energy Systems: Analysis of Technical \& Economic Issues

prepared by Giovanni Maronati, Roberto Ponciroli, and Richard B. Vilim

Nuclear Engineering Division, Argonne National Laboratory

August 30, 2018 


\section{Abstract}

Nuclear-Renewable Hybrid Energy Systems (N-R HES) combine different energy technologies in a synergistic way with the objective of achieving a more economical energy production. In this report the particular hybrid system dealt with includes a PWR nuclear reactor, the associated Rankine-based energy conversion cycle (Balance of Plant, BOP), an Industrial Process (IP) for hydrogen production, a gas turbine (Second Energy Source, SES), and a battery (Energy Storage, ES). The RAVEN-based HYBRID framework is used to find the optimal installed capacity and the optimal economical dispatch of each component of the N-R HES. HYBRID solves the coupled capacity planning-economic and dispatch optimization problem with the objective of minimizing the Levelized Cost of Electricity (LCOE).

In the optimization of the power dispatch strategy, the HYBRID framework takes into account the time-dependent dynamics of each subsystem by including a Dymola object-oriented model of the process dynamics. To ease the computational burden of the optimization process, an initial guess for the dispatch optimization problem is provided by a preconditioner. The solution guess is calculated by means of a least-marginal cost approach which applies the same criterion behind the screening curve method. Though this algorithm allows evaluating an economically adapted generation mix for a target load duration curve within a few seconds, it neglects the constraints due to the single component dynamics, and it is not able to adequately represent the ES.

An improved treatment of the pre-conditioner problem was addressed by introducing a Monte Carlo based optimization algorithm. The new approach takes into account limits on power rates for the system subcomponents, which was not previously done. Additionally, this new MonteCarlo based preconditioner addresses the problem with the ES component through improved modeling of that component. The new algorithm has been implemented in the HYBRID framework and tested to assess its performance. The results obtained with the new Monte Carlobased preconditioner were benchmarked against those obtained through the reference deterministic approach. The comparison shows that the Monte-Carlo based approach provides improved solution accuracy to the power dispatch optimization problem. Also in this report, the treatment of the penalty function on LCOE is discussed, and an improved penalty function for missed demand is suggested and tested. 


\section{Table of Contents}

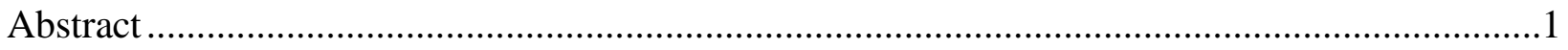

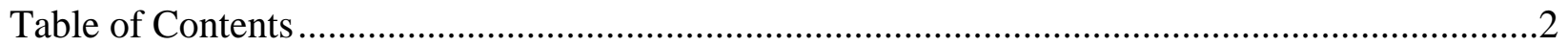

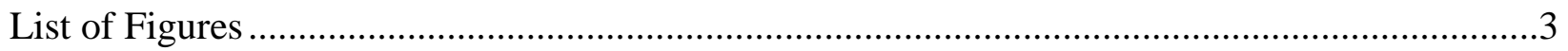

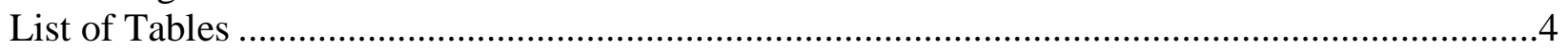

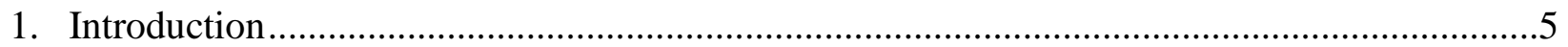

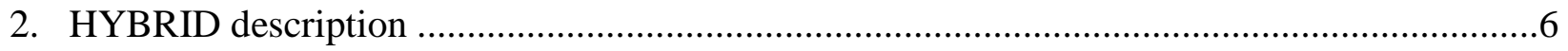

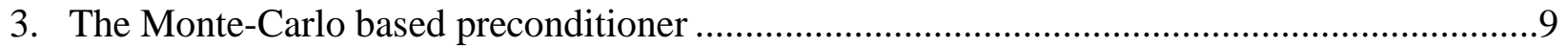

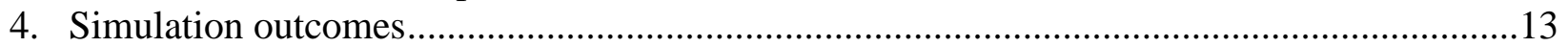

4.1. Reference case: 300,000 scaling demand ...........................................................

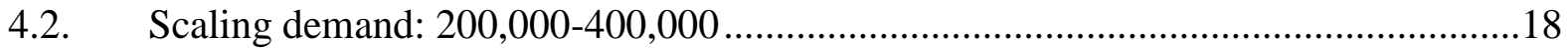

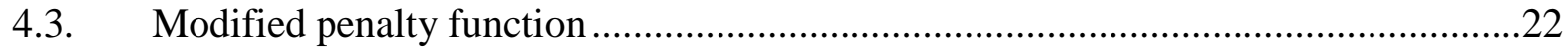

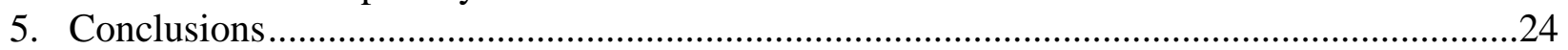

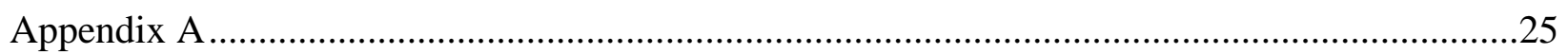

A1. Preconditioner implementation in HYBRID .....................................................2.

A.1.1. Preconditioner in the RAVEN outer level.............................................................25

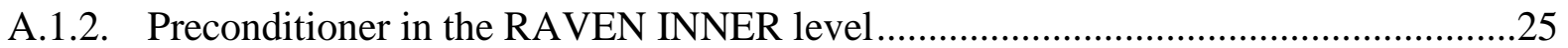

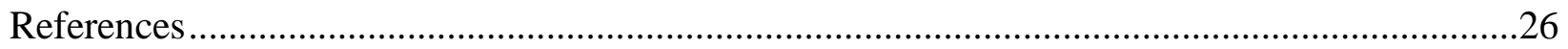




\section{List of Figures}

Figure 1 - Schematic of the HYBRID framework for capacity planning and dispatch optimization

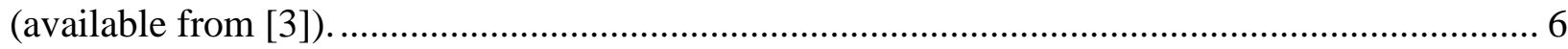
Figure 2 - Schematic of the HYBRID framework for capacity planning and dispatch optimization (simplified). 7

Figure 3 - Penalty function for missed demand (overproduction and underproduction)............... 8

Figure 4 - Graphical representation of the BOP and IP power consumptions ............................... 9

Figure 5 - Graphical representation of the implemented criterion ............................................... 10

Figure 6 - Conceptual steps of the ANL preconditioner algorithm............................................. 11

Figure 7 - Schematic of the HYBRID framework, modified to include the Monte Carlo-based

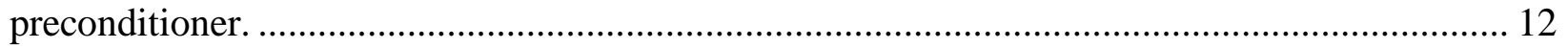

Figure 8 - Total power and net demand as a function of time (300,000 scaling demand) ........... 14

Figure 9 - BOP and SES dispatched power as a function of time (300,000 scaling demand) ..... 15

Figure 10 - IP electric power and ES level as a function of time (300,000 scaling demand) ...... 15

Figure 11 - LCOE minimum as a function of the iteration number ............................................ 16

Figure 12 - Total power for different iterations and net demand as a function of time ................ 17

Figure 13 - Fraction of total system costs (discounted) …….................................................... 17

Figure 14 - Total power and net demand as a function of time (200,000 scaling demand) ......... 18

Figure 15 - BOP (a) and SES (b) dispatched power as a function of time (200,000 scaling demand)

Figure 16 - IP electric power (a) and ES level (b) as a function of time (200,000 scaling demand)

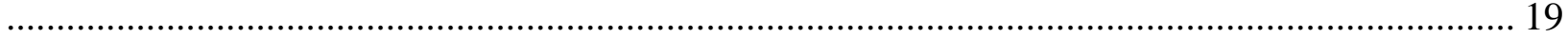

Figure 17 - Total power and net demand as a function of time (400,000 scaling demand)......... 20

Figure 18 - BOP (a) and SES (b) dispatched power as a function of time (400,000 scaling demand)

Figure 19 - IP electric power (a) and ES level (b) as a function of time (400,000 scaling demand)

Figure 20 - LCOE minimum as a function of the iteration number .......................................... 21

Figure 21 - Modified penalty function for missed demand........................................................ 22

Figure 22 - Fraction of total system costs (discounted) (modified penalty function).................. 23 


\section{List of Tables}

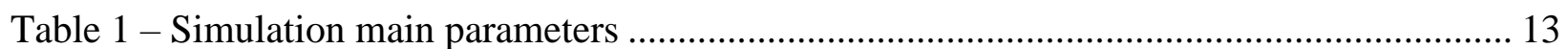

Table 2 - LCOE minimum as a function of the iteration number .......................................... 16

Table 3 - LCOE as a function of the scaling demand (INL vs ANL preconditioner) ................. 18

Table 4 - LCOE as a function of the scaling demand (modified penalty function).................... 22 


\section{Introduction}

A RAVEN-based framework, called HYBRID, was developed to perform economic evaluations of N-R HES (Nuclear-Renewables Hybrid Energy Systems) and presented in [1]. One of the goals of the HYBRID modeling and simulation project is to assess the economic viability of hybrid systems in a market with a high penetration of renewable energy sources. The investigated configuration of N-R HES includes a PWR-type nuclear reactor, the associated Rankine-based energy conversion cycle (Balance of Plant, BOP), an Industrial Process (IP) for hydrogen production, a gas turbine (Second Energy Source, SES), and a battery (Energy Storage, ES). The nuclear reactor both generates electricity to supply the grid and provides heat/electricity to the IP. In the current case study, the IP produces hydrogen but other processes, such as water desalinization, are under consideration. The coordination of energy suppliers characterized by very different dynamics to meet load demands is the key ingredient to provide lower electricity costs and deal with cost volatility. The analysis of this system includes both an optimization of the sizing of the components/subsystems of the N-R HES (capacity planning) and the optimal dispatch strategy by all components to minimize the Levelized Cost of Electricity (LCOE).

The software framework to solve the capacity planning and energy dispatch problems for the N-R HES was introduced in [2][3]. The solution of the coupled problem, in order to be feasible, needs to satisfy the dynamic constraints for all the system components. The system dynamic response is evaluated by means of a dedicated Dymola object-oriented model. However, to lower the computational burden of the simulations, a guess solution of the dispatch problem needs to be provided. To this aim, a "pre-conditioner" based on least-marginal cost approach was developed [2][3]. However, the proposed approach does not account for limits on ramp rates, and therefore is not guaranteed to provide a sufficiently accurate guess solution. Furthermore, the initial dispatch is calculated through a deterministic approach which resembles the screening curve method. This approach is based on a fixed logic that prioritizes electricity production through the gas turbine over the use of stored energy in the battery, and does not guarantee the solution optimality.

The approach presented in [4] tries to address the main weaknesses of this approach by describing the capabilities of a pre-conditioner algorithm which takes into account the dynamics-derived operational constraints on the power ramp rates. This algorithm provides an initial dispatch solution through Monte Carlo sampling. This approach better ensures that an initial guess is found that is in the neighborhood of the actual solution [5]. In this work, the modeling of the ES operation strategy was improved, and the Monte Carlo-based preconditioner was implemented in the HYBRID framework. The algorithm was then tested and compared to the reference deterministic preconditioner.

This report is structured as follow. Section 2 describes the structure of the HYBRID framework. The third section describes the proposed approach to provide the initial dispatch through a MonteCarlo based preconditioner. The fourth section shows the simulations that were performed to test and compare the presented approach (the ANL preconditioner) against the reference one (the INL preconditioner). Finally, the main outcomes presented in this work and areas for further enhancement are briefly summarized in the conclusions. 


\section{HYBRID description}

HYBRID is a software framework based on RAVEN modules that was developed to perform economic statistical analysis and optimization of N-R HES. The framework allows analysis and optimization of both capacity planning and dispatch problems, as described in detail in [2][3]. The complete schematic of the framework is shown in Figure 1. For ease of reading, a simplified schematic of the RAVEN modules is shown in Figure 2, which shows the main interconnections between the RAVEN modules.

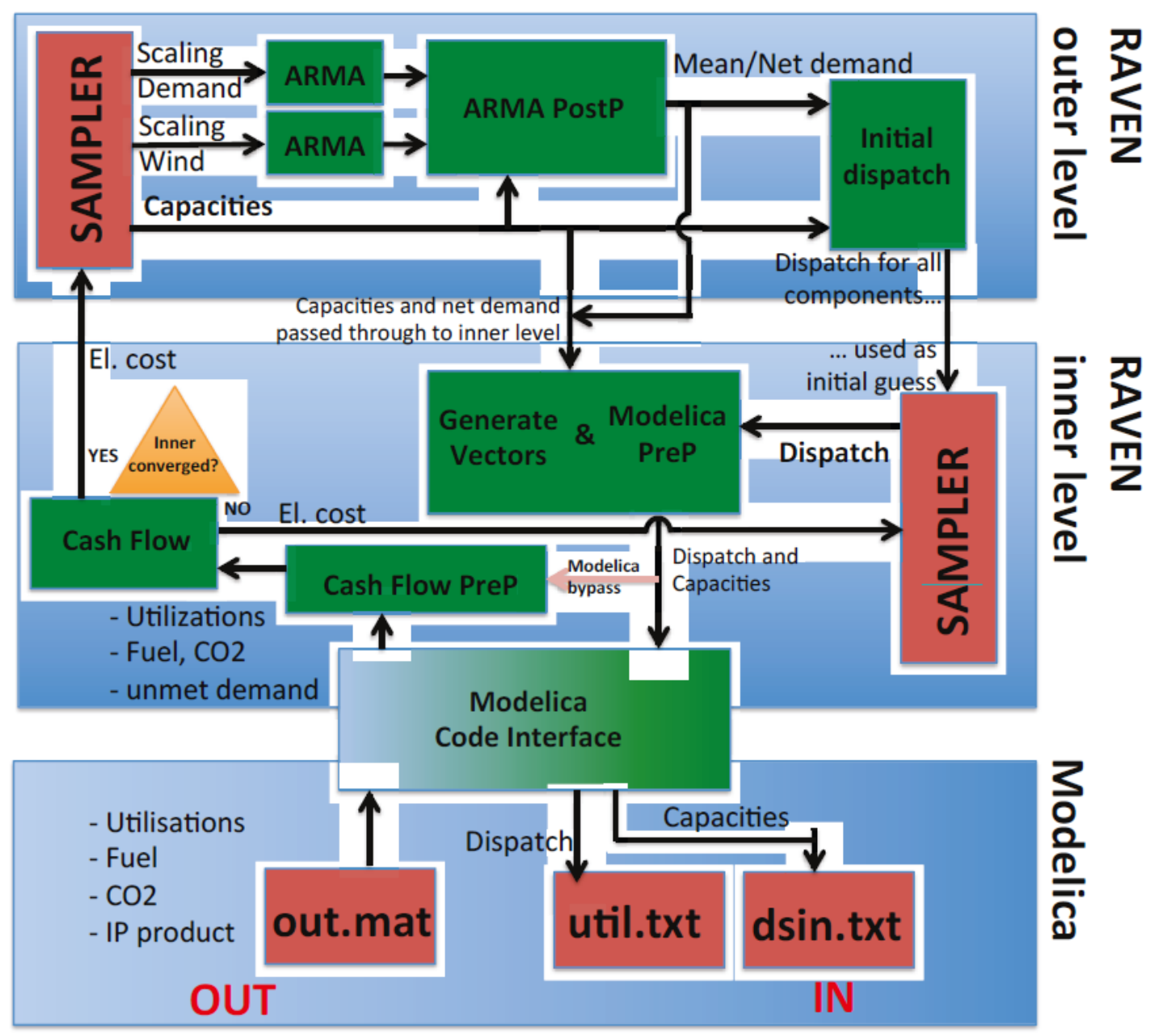

Figure 1 - Schematic of the HYBRID framework for capacity planning and dispatch optimization (available from [3]). 


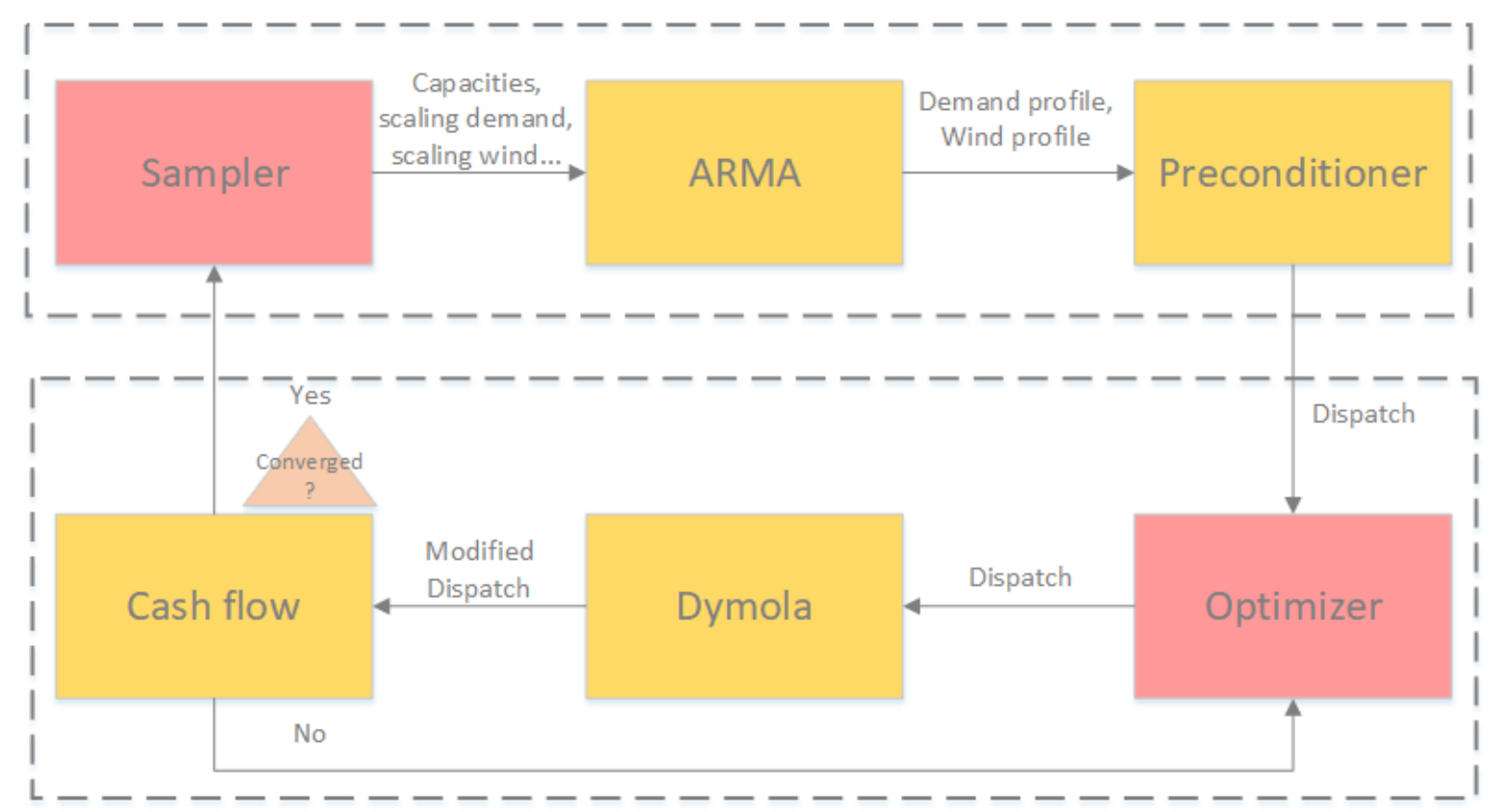

Figure 2 - Schematic of the HYBRID framework for capacity planning and dispatch optimization (simplified).

HYBRID is made of a RAVEN outer level and a RAVEN inner level. In a simulation, the outer level of RAVEN is run first, and it recalls another instance of RAVEN (the inner level) inside the simulation. The outer level has samples the capacities of all components of the N-R HES, as well as the scaling demand and the renewable penetration. The renewable penetration $\left(R E N_{\text {fract }}\right)$ is defined as the installed capacity percentage of renewable sources $\left(P_{R E N}\right)$ over the total installed capacity of all electricity sources $\left(P_{B O P}+P_{S E S}+P_{R E N}\right)$ :

$$
R E N_{\text {fract }}=\frac{P_{R E N}}{P_{B O P}+P_{S E S}+P_{R E N}}
$$

In the studied problem, renewable energy is only produced by wind turbines. The scaling demand is the factor that multiplies the electricity demand and used to train the ARMA. The training demand has a mean value of about $1.1 \mathrm{~kW}$. Therefore, the scaling demand "almost" represents the mean value of the demand. For example, a scaling demand of 200,000 represents an actual demand with mean value of about $220 \mathrm{MW}$. The outer level sampler can be a grid sampler, a Monte Carlo sampler, or an optimizer. The sampled parameters are then passed to the ARMA module, which samples the electricity demand and the wind speed profile. The wind speeds are then converted into the electricity production profile from wind in an ARMA post-processor (not shown in the schematic), which also calculates the demand, "net" of renewable production. The net demand, which is the difference between the gross demand and the wind production, is fed to the preconditioner module together with all the other sampled parameters. The use of the net demand in the dispatch assessment ensures that all electricity produced through renewable sources is dispatched first, while all the other components take care of the remaining demand. The preconditioner (or initial dispatch) module developed by INL and presented in [4] relies on a deterministic approach which sorts the units to be committed by adopting the same criterion behind 
the screening curve method, i.e. the generating units characterized by lower marginal costs are used first. This approach is widely used, as it allows evaluating an economically adapted generation mix for a target load duration curve within a few seconds. However, it neglects the constraints due to the single component dynamics, and it can hardly be modified to account for the presence of the ES. In addition, this module was developed with a specific dispatch order (or logic), where the marginal cost is known a priori and are specific to the case. In case the cost inputs are changed, the module needs to be suitably modified to reflect the new dispatch order, based on the new marginal costs.

The dispatch resulting from the dispatch module is sent to the inner level of RAVEN. In the inner level, the initial dispatch is assumed to be optimal and is sent to the Dymola model of the N-R HES unit. This module takes as input the power output profile derived by the preconditioner and simulates the corresponding controlled dynamic response. The ensuing power profiles are eventually sent to the Cash Flow module, which evaluates the corresponding cash flow and calculates the cost metric (LCOE, NPV, or IRR). The Cash Flow module analyzes the marginal costs based on the dispatch, and together with the fixed costs calculates the cost metric. In particular, a certain penalty is foreseen to discourage underproduction and overproduction scenarios. The penalty function is expressed by Eq. (2) and is plotted in Figure 3. In the equation, $P_{\text {dem }}$ represents the net demand at time $t, P_{\text {disp }}$ represents the dispatched power at time $t$. The function is saturated at $\$ 400 / \mathrm{MWh}$, i.e. for values of the function higher than $\$ 400 / \mathrm{MWh}$, the function is set to the saturation value.

$$
\text { Penalty }\left(\frac{\$}{M W h}\right)=12 \cdot 0.4699 \cdot \exp \left(1.0141 \cdot\left|P_{\text {dem }}(M W h)-P_{\text {disp }}(M W h)\right| / 12\right)
$$

The dispatch and the cost metric are sent to the inner level optimizer, which iterates attempts to optimize the cost metric by changing the dispatch until the convergence criteria are satisfied or it reaches the maximum number of iterations.

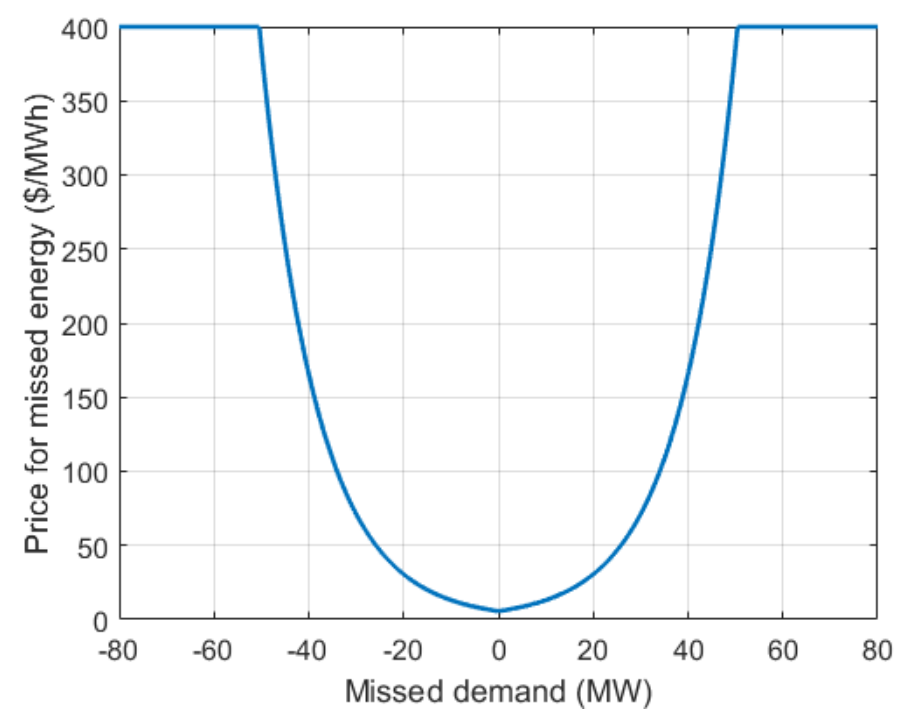

Figure 3 - Penalty function for missed demand (overproduction and underproduction). 


\section{The Monte-Carlo based preconditioner}

Unlike the INL marginal-cost based preconditioner, the ANL Monte Carlo-based preconditioner includes power drop and power rise ramp rate limits for both the BOP and the SES, derived from the Dymola model [4][5]. In particular, the instantaneous power outputs $\left(P_{i}(t)\right)$ for BOP and SES are calculated from the power outputs at the previous time step $\left(P_{i}(t-1)\right)$ through Eq. (3). The power variation of a component at time $t$ is calculated by multiplying the sampled random number $(\operatorname{rand}(-1,1))$ by the corresponding maximum sustainable power variation $\left(\Delta P_{i}^{\max }\right)$. In this way, the operational constraints are inherently met, i.e., the sampled power profiles do not violate the limits imposed by the characteristic ramp-rate constraints.

$$
P_{i}(t)=P_{i}(t-1) \cdot \operatorname{rand}(-1,1) \cdot \Delta P_{i}^{\max }
$$

The leftover thermal power produced by the nuclear reactor is used by the IP. The IP level is calculated as the difference between the nuclear reactor total power production and the BOP power (Eq. (4)) [6].

$$
P_{I P}(t)=P_{e l, I P}(t)+P_{t h, I P}(t) \cdot \eta=P_{e l, I P}(t)+\frac{P_{e l, I P}(t)}{2.77} \cdot \eta
$$

Figure 4 shows how the thermal power produced by the nuclear reactor is shared by the BOP and the IP when the IP is at full capacity. Hydrogen production needs electric power and thermal power from with a ratio of 2.77. When the IP operates at full capacity, to an installed capacity of 51.17 $\mathrm{MW}_{\mathrm{el}}$ corresponds a thermal consumption of $18.47 \mathrm{MW}_{\text {th }}$. The remaining power available to the BOP for electricity production is:

$$
P_{I P}(t)=51.17 M W_{e l}+18.47 M W_{t h} \cdot 0.31 \frac{M W_{e l}}{M W_{t h}}=243.10 M W_{e l}
$$

where $P_{e l, I P}$ and $P_{t h, I P}$ represent the IP electric and thermal power consumptions, and $\eta$ is the thermal BOP efficiency [6].

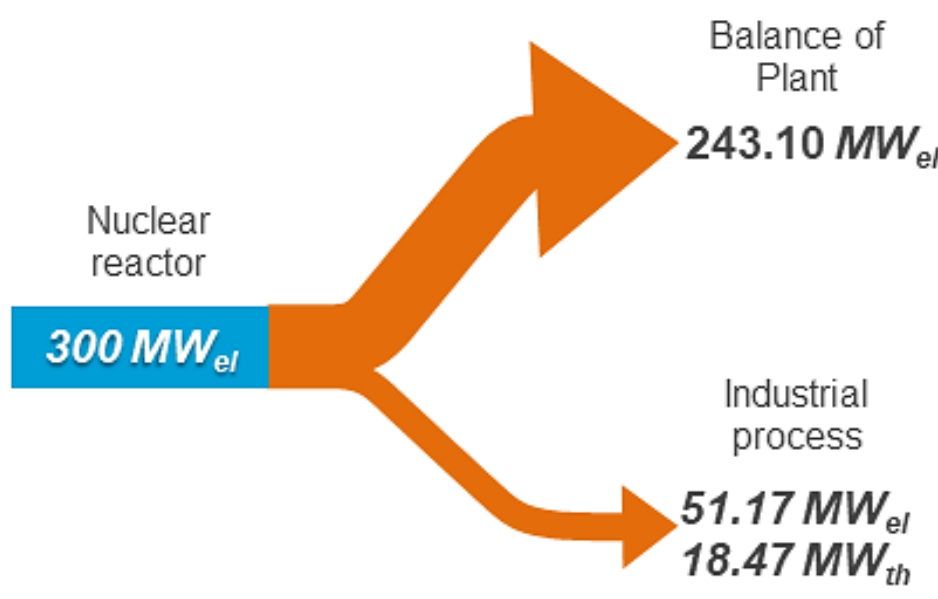

Figure 4 - Graphical representation of the BOP and IP power consumptions 
The sum of the BOP and SES power contributions is then compared to the net demand, and the ES level is updated according to Eq. (6). If the difference between the net demand and produced power is negative, the ES is in "charge" mode, while if it is positive, the ES is in "discharge" mode. The outgoing power of the ES is the difference between the net demand and the power produced by the BOP and the SES, within the limits imposed by the ES capacity. The energy level of the ES $(E(t))$ is then the difference between the energy level $(E(t-1))$ and the outgoing power $(P(t-1))$ in the previous time step (Figure 5). Under this approach, the ES outgoing power is assumed constant in the time step (1 hour). Eq. (7) represents the ES level calculation at the first time step.

$$
\begin{aligned}
& E(t)=E(t-1)-P(t-1) \cdot 1 h \\
& E(1)=E(0)-P(0) \cdot 1 h
\end{aligned}
$$

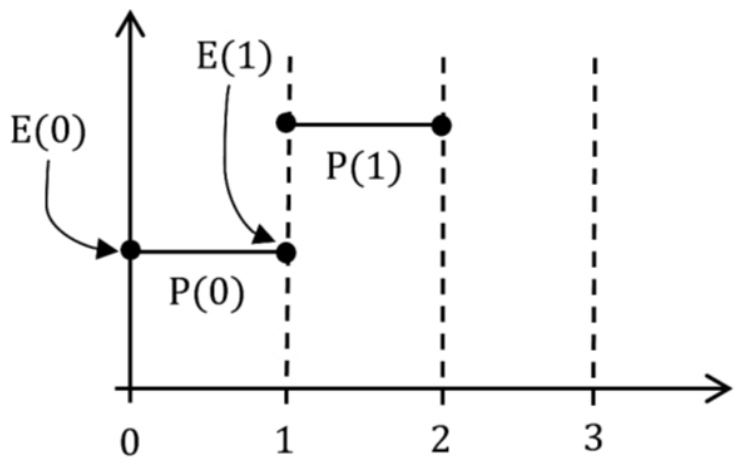

Figure 5 - Graphical representation of the implemented criterion

In this process, the ES capacity bounds need to be respected. If the ES level calculated through Eq. (6) is higher than the ES capacity, the ES level is saturated with the value of ES installed capacity. This case represents the scenario in which the ES is full. In case the ES level calculated through Eq. (6) is negative, the ES level is set to zero. This implies that the net demand is not fully satisfied, which will in result in a missed demand.

All instantaneous power outputs of all the components are then adjusted to meet the net demand through normalization. In particular, it is unlikely that sampling power rates from a uniform distribution will satisfy the net demand at each dispatch hour. The following normalization process of the power outputs of each component increases the likelihood that the net demand will be satisfied, and reduces the chances that an over-production or an under-production penalty will be paid. The power outputs of each component $P(i, t)$ are normalized according to Eq. (8) [5], where $M$ is the number of generating electricity components of the N-R HES.

$$
P(i, t)=P(i, t) \frac{N e t D(t)}{\sum_{t=1}^{M} P(i, t)}
$$

Since through normalization all the power outputs are altered, it might happen that constraints are violated in the end ramp rate. Therefore, after normalization, all operational constraints are verified. If the limitations on both power increases and power drops are not met, the sampled 
values are saturated. Finally, a last saturation effect is imposed to respect the maximum/minimum power bounds. A summary of the preconditioner algorithm steps is shown in Figure 6.

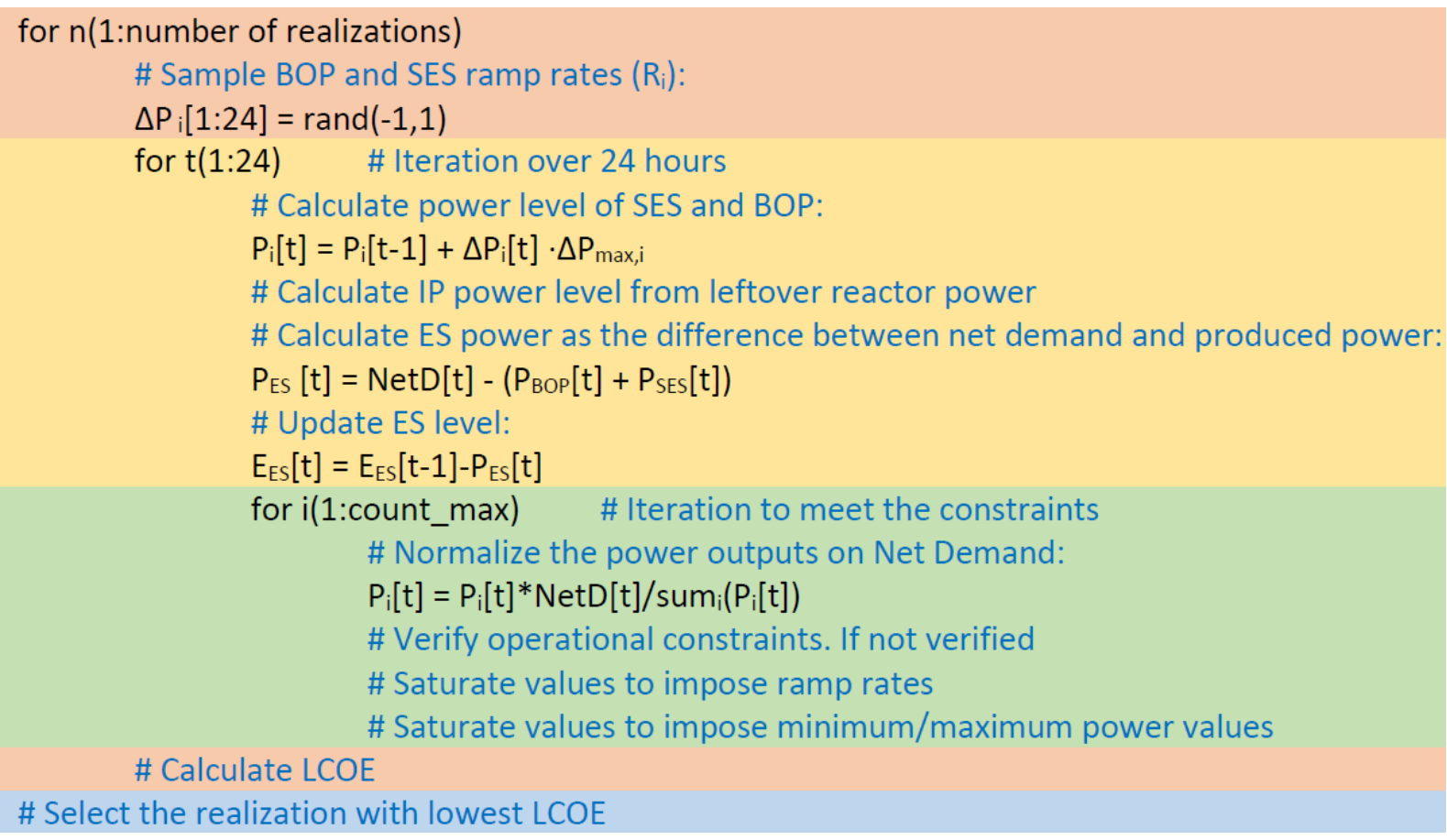

Figure 6 - Conceptual steps of the ANL preconditioner algorithm.

The Monte Carlo-based preconditioner was initially implemented in the RAVEN outer level. The hourly power ramps for the BOP and the SES were sampled through a Monte Carlo sampler. However, the computational time required to perform a simulation was considerable (about 100,066 seconds for 10,000 iterations). Following the advice of the RAVEN developers, Monte Carlo sampler and the preconditioner were then moved to the inner level of RAVEN. A schematic of the modified HYBRID model is shown in Figure 7.

The hourly dispatch produced by the preconditioner is then evaluated by the Cash Flow module, which calculates the LCOE of the system over its lifetime. The 24-hour dispatch period is used for all days during the lifetime of the system. This is a strong approximation, which does not represent a realistic scenario. However, this method is valid for the purpose of comparing the INL deterministic approach to the ANL Monte Carlo based approach. The LCOE values are collected and evaluated by a Post Processor module, which picks the one with lowest LCOE. The corresponding Monte Carlo realization is finally passed to the Dymola module. 


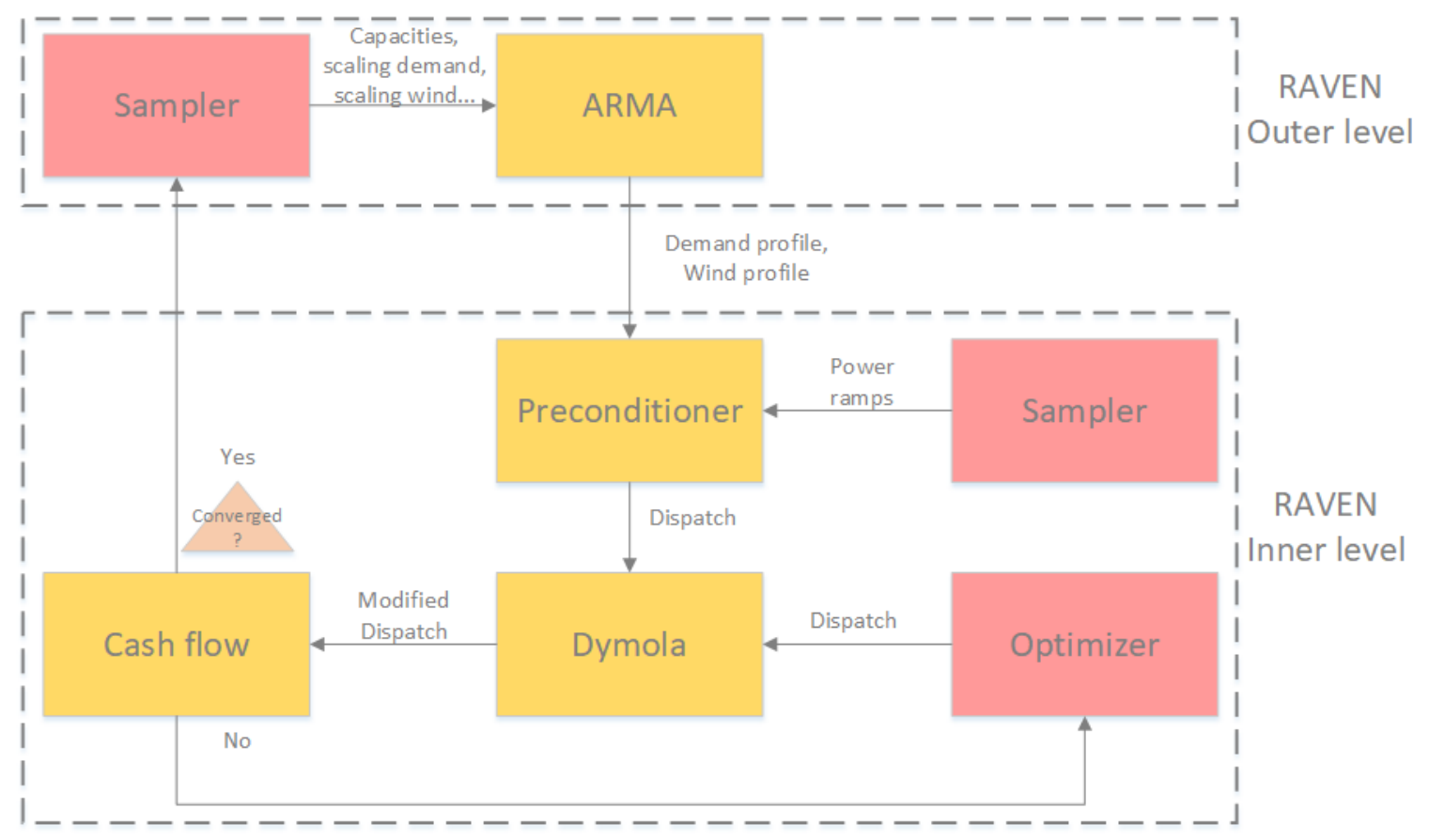

Figure 7 - Schematic of the HYBRID framework, modified to include the Monte Carlobased preconditioner.

Few modifications were made to the preconditioner presented in [4][5]. The ES operation model expressed by Eq. (6) was implemented. The cash flow module was developed to calculate the variable O\&M costs based on the equivalent instantaneous electric power level of the reactor. The equivalent instantaneous electric power is defined as the electric power dispatched to the grid or stored in the ES, the electric power transferred to the IP, and the electric-equivalent of the thermal power transferred to the IP. The first version of the preconditioner was set to give as output the reactor electric power delivered to the grid, and not the total equivalent electric power produced by the reactor. The O\&M cost and fuel costs were then underestimated, as they were calculated from the BOP electric power, despite the nuclear reactor works at full capacity. The preconditioner was modified to output the equivalent electric power. However, under this approach, the variable O\&M cost is treated as a fixed cost, as the nuclear reactor is operated at full power capacity. Furthermore, this case assumes that all the variable O\&M cost is associated with the primary system, and not to the O\&M of the balance of plant. A distinction between primary system O\&M cost and BOP O\&M cost will be addressed in future work. 


\section{Simulation outcomes}

Simulations were run with the parameters shown in Table 1. The comparison between INL and ANL preconditioners was done for values of the scaling demand of 200,000, 300,000, and 400,000. 10,000 iterations were run for the ANL preconditioner. The renewable penetration was set to $0 \%$ to decrease the volatility of the net demand and be consistent with the net demand profile between all benchmark cases. Furthermore, as shown by [7], for the cost inputs currently used in the HYBRID, the optimal system configuration is for a $0 \%$ renewable penetration. LCOE was calculated using a Weighted Average Cost Of capital (WACC) of 5\%, an inflation rate of 3\%, and a tax rate of $39.2 \%$. A summary of what cash flows are affected by inflation and tax rate is presented in [3].

Table 1 - Simulation main parameters

\begin{tabular}{|c|c|c|c|}
\hline & & INL & ANL \\
\hline Scaling demand & $\begin{array}{l}200,000 \\
300,000 \\
400,000\end{array}$ & $\mathrm{X}$ & $\mathrm{X}$ \\
\hline BOP capacity & $300 \mathrm{MW}$ & $\mathrm{X}$ & $\mathrm{X}$ \\
\hline SES capacity & $35 \mathrm{MW}$ & $\mathrm{X}$ & $\mathrm{X}$ \\
\hline $\begin{array}{l}\text { IP nominal electricity } \\
\text { usage }\end{array}$ & $51.17 \mathrm{MW}$ & $\mathrm{X}$ & $\mathrm{X}$ \\
\hline IP nominal heat usage & $18.48 \mathrm{MW}$ & $\mathrm{X}$ & $\mathrm{X}$ \\
\hline ES capacity & $20 \mathrm{MWh}$ & $\mathrm{X}$ & $\mathrm{X}$ \\
\hline Renewable penetration & $0 \%$ & $\mathrm{X}$ & $\mathrm{X}$ \\
\hline BOP efficiency & 0.31 & $\mathrm{X}$ & $\mathrm{X}$ \\
\hline ES time constant & $4 \mathrm{~h}$ & $\mathrm{X}$ & $\mathrm{X}$ \\
\hline $\mathrm{H}_{2}$ price & $1.75 \$ / \mathrm{kg}$ & $\mathrm{X}$ & $\mathrm{X}$ \\
\hline Nominal $\mathrm{H}_{2}$ production & $0.40 \mathrm{~kg} / \mathrm{s}$ & $\mathrm{X}$ & $\mathrm{X}$ \\
\hline$P_{B O P}^{\max }$ & $300 \mathrm{MW}$ & $\mathrm{X}$ & $\mathrm{X}$ \\
\hline$P_{B O P}^{\min }$ & $139.5 \mathrm{MW}$ & $\mathrm{X}$ & $\mathrm{X}$ \\
\hline$P_{S E S}^{\max }$ & $35 \mathrm{MW}$ & $\mathrm{X}$ & $X$ \\
\hline$P_{S E S}^{\min }$ & $13.125 \mathrm{MW}$ & $X$ & $\mathrm{X}$ \\
\hline$P_{I P}^{\max }$ & $51.17 \mathrm{MW}$ & $X$ & $X$ \\
\hline$P_{I P}^{\min }$ & $0 \mathrm{MW}$ & $\mathrm{X}$ & $\mathrm{X}$ \\
\hline$\Delta P_{B O P}^{\max }$ & $55 \mathrm{MW}$ & & $\mathrm{X}$ \\
\hline$\Delta P_{B O P}^{\min }$ & $-55 \mathrm{MW}$ & & $\mathrm{X}$ \\
\hline$\Delta P_{S E S}^{\max }$ & $15 \mathrm{MW}$ & & $\mathrm{X}$ \\
\hline$\Delta P_{S E S}^{\min }$ & $-29 \mathrm{MW}$ & & $\mathrm{X}$ \\
\hline
\end{tabular}

The simulations through the HYBRID framework presented in [3] were run with a storage at full capacity as initial condition. However, as shown in [7], the initial condition might impact the validity of the results. Therefore, in this set of simulations, the ES initial energy level was set to 
zero. If the ES is initially fully charged, the ANL preconditioner would favor the storage over the other components, since its variable O\&M costs are virtually zero. This represents a problem in case the simulated timeframe is extrapolated to represent each day of the lifetime. This case is equivalent to using the same net demand and dispatch profile every day of the system lifetime. Under this conditions, the ANL preconditioner would pick the realization where the ES is emptied to satisfy the net demand, without being replenished by the end of the day. As the following day is simulated through the same dispatch (ES full as initial condition), the conservation of energy is violated. Having an empty storage as initial condition does not automatically imply that energy is conserved, as the energy is not conserved if the ES has some energy stored at the end of the day. However, this initial condition represents a more conservative scenario, where the energy stored at the end of the day is "wasted".

\subsection{Reference case: 300,000 scaling demand}

The dispatch profiles for a scaling demand of 300,000 obtained by the INL and ANL preconditioners are shown in Figure 8, along with the imposed net demand profile. The hourly power profiles for all components are shown in Figure 9a, Figure 9b, and Figure 10a, while the ES level is shown in Figure 10b. The LCOE obtained by the INL preconditioner is $\$ 192.14 / \mathrm{MWh}$ while, for the ANL preconditioner, the LCOE minimum at the 5,239 ${ }^{\text {th }}$ iteration, and is equal to $\$ 121.29$ /MWh. This value is $36.87 \%$ lower than that obtained by the INL preconditioner.

The IP power profile obtained through the ANL preconditioner is lower between hours 5-12, while the ES storage level is always equal or greater (Figure 10). In the ANL scenario, the battery is prioritized over the IP. Having a higher amount of stored energy during the demand peak (hours 11-16) allows the system to make a lesser use of the SES, which is the component characterized by the highest marginal cost (Figure 9). Furthermore, this dispatch allows a better matching between the power production and the net demand, than the one obtained through the INL preconditioner between hours 11-16 (Figure 8), with a subsequent lower penalty for missed demand.

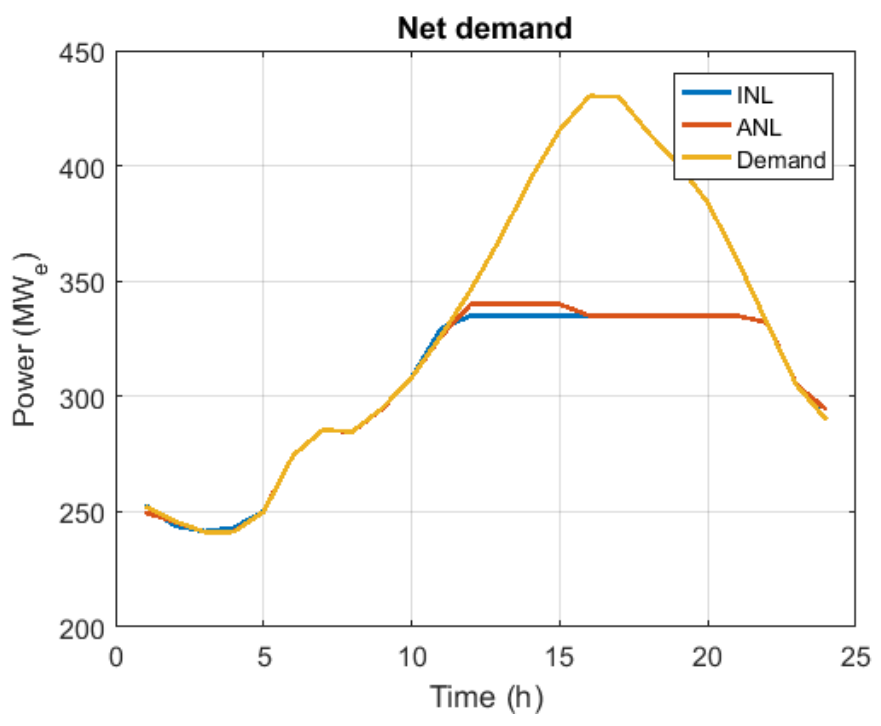

Figure 8 - Total power and net demand as a function of time (300,000 scaling demand) 


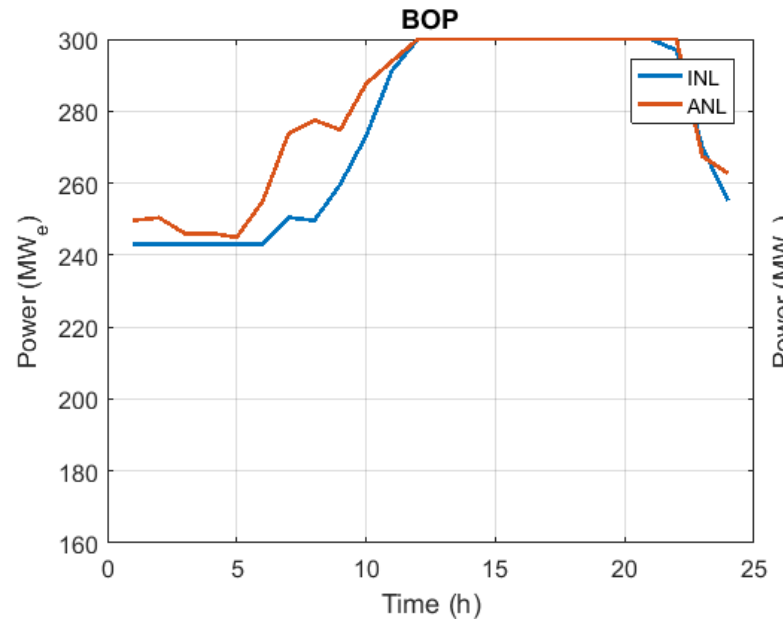

(a)

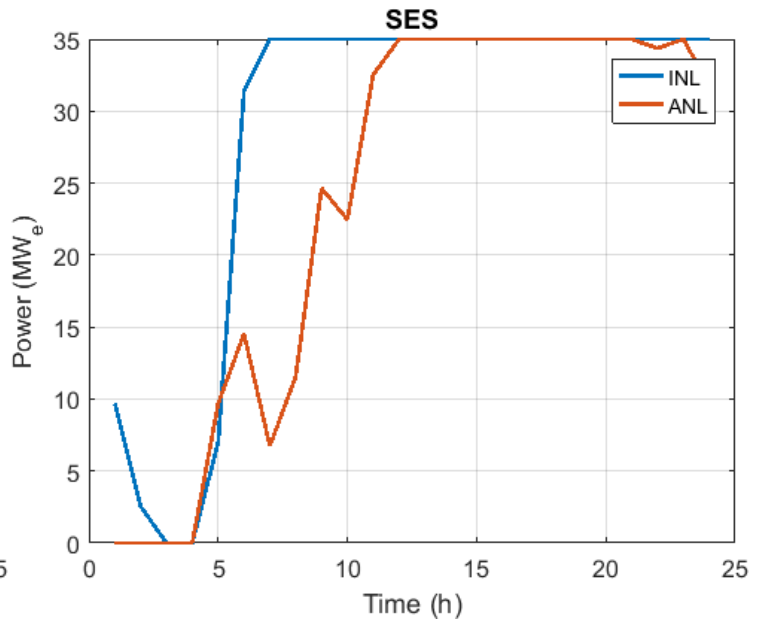

(b)

Figure 9 - BOP and SES dispatched power as a function of time (300,000 scaling demand)

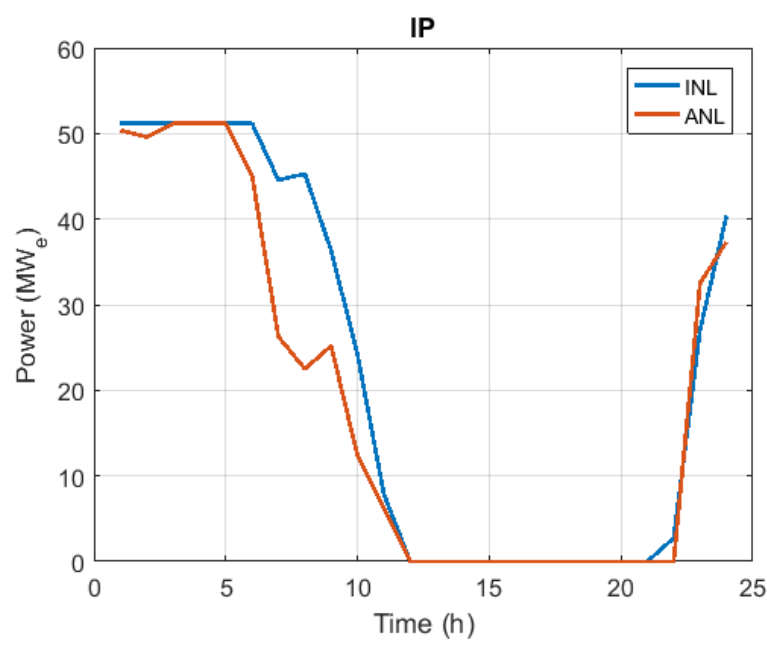

(a)

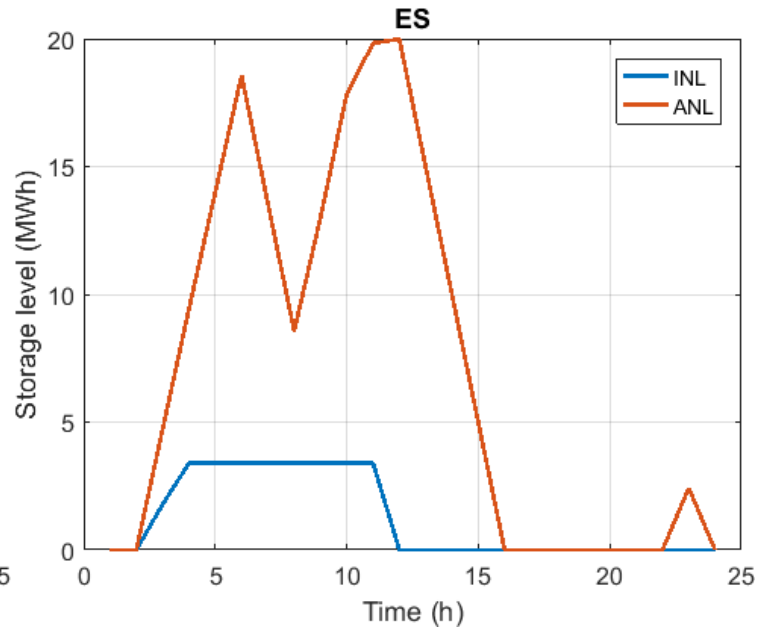

(b)

Figure 10 - IP electric power and ES level as a function of time (300,000 scaling demand)

The INL preconditioner follows a deterministic method, which is based on a fixed priority logic. The power dispatch algorithm is based on the marginal costs of the components, i.e., components with lower marginal costs are dispatched first. The algorithm only considers the present state of the system (net demand profile, power values), without taking into account the future behavior of the net demand. INL preconditioner algorithm is based on the following assumptions:

1. In case of overproduction, the IP has to priority over the battery to absorb power;

2. The ES is always last in the dispatch logic, i.e. the energy stored in the battery is always dispatched last.

From the first assumption follows that the algorithm does not take into account that hydrogen production is not profitable at all times. In fact, for prices of hydrogen lower than the IP marginal 
cost, hydrogen production is not economically convenient. Furthermore, this assumption implies that the ES cannot be used at times when it is most needed. This assumption, together with the second assumption, implies that the ES cannot be used for production before the SES, even if its marginal costs are lower than those of the SES. In some cases, pre-charging the battery to meet future demand peaks could be more profitable than producing hydrogen and using the SES (which is notably characterized by high marginal costs) in the future.

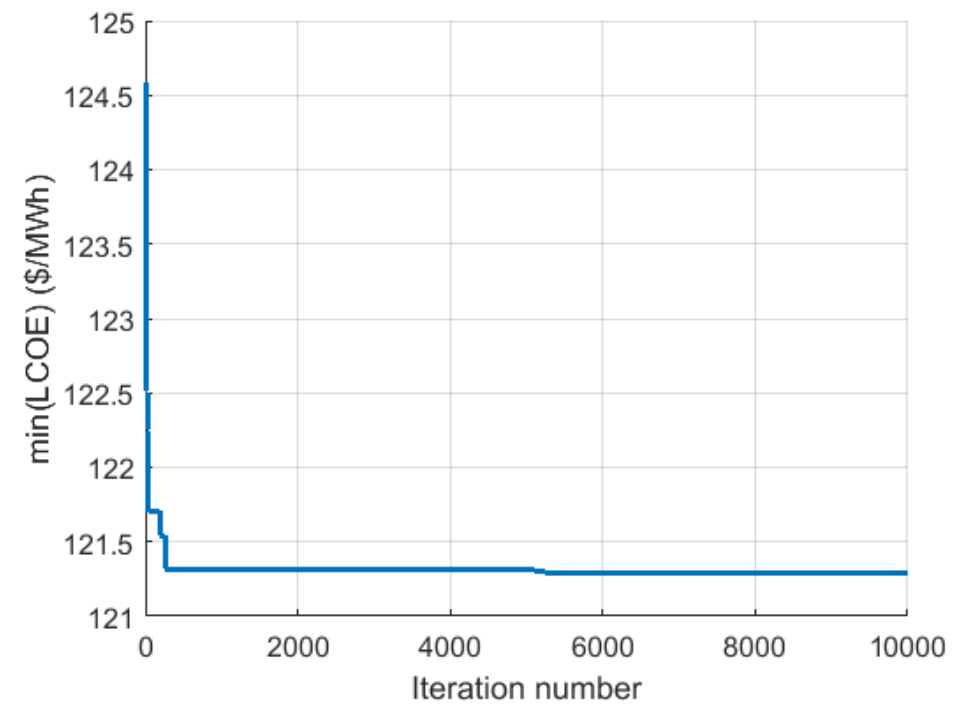

Figure 11 - LCOE minimum as a function of the iteration number

Table 2 - LCOE minimum as a function of the iteration number

\begin{tabular}{ll}
\hline Iteration & $\begin{array}{l}\text { Min(LCOE) } \\
(\$ / M W h)\end{array}$ \\
\hline 1 & 124.59 \\
\hline 2 & 122.64 \\
\hline 7 & 122.51 \\
\hline 13 & 122.50 \\
\hline 27 & 122.27 \\
\hline 32 & 122.24 \\
\hline 43 & 121.70 \\
\hline 196 & 121.53 \\
\hline 5,116 & 121.32 \\
\hline 5,239 & 121.31 \\
\hline
\end{tabular}

The LCOE minimum between the $1^{\text {st }}$ and the $n^{\text {th }}$ iteration as a function of the iteration number is plotted in Figure 11. The values are shown in Table 2. It is important to note that at the second iteration LCOE $\$ 122.64 / \mathrm{MWh}$, only $1.1 \%$ higher than the minimum for the whole simulation, which is obtained at iteration 5,239. The total production of all components for iteration 2, 32, and 5,239 are shown in Figure 12, along with the net demand profile. The plot shows that the power profile matches the net demand very quickly. 
For the case resulting with minimum LCOE, the LCOE calculation was performed outside RAVEN to assess the cost fractions of all cost factors. The results of the calculation are shown in Figure 13. The highest cost is the BOP capital cost (37.2\% of LCOE), followed by the penalty for missed demand (27.0\%), and the BOP O\&M cost (14.0\%). The other costs make up the remaining percentage. As the penalty represents a higher portion of the total negative cash flow, a proper modeling of the cost function is needed. The cost function was modified to represent a more realistic scenario, as shown in Section 4.4.

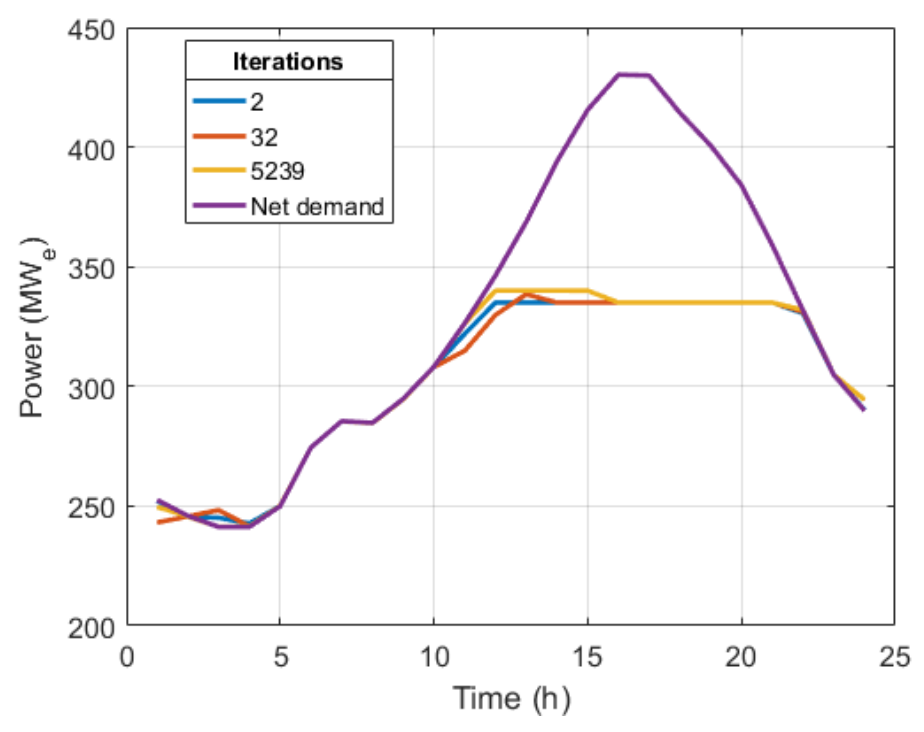

Figure 12 - Total power for different iterations and net demand as a function of time

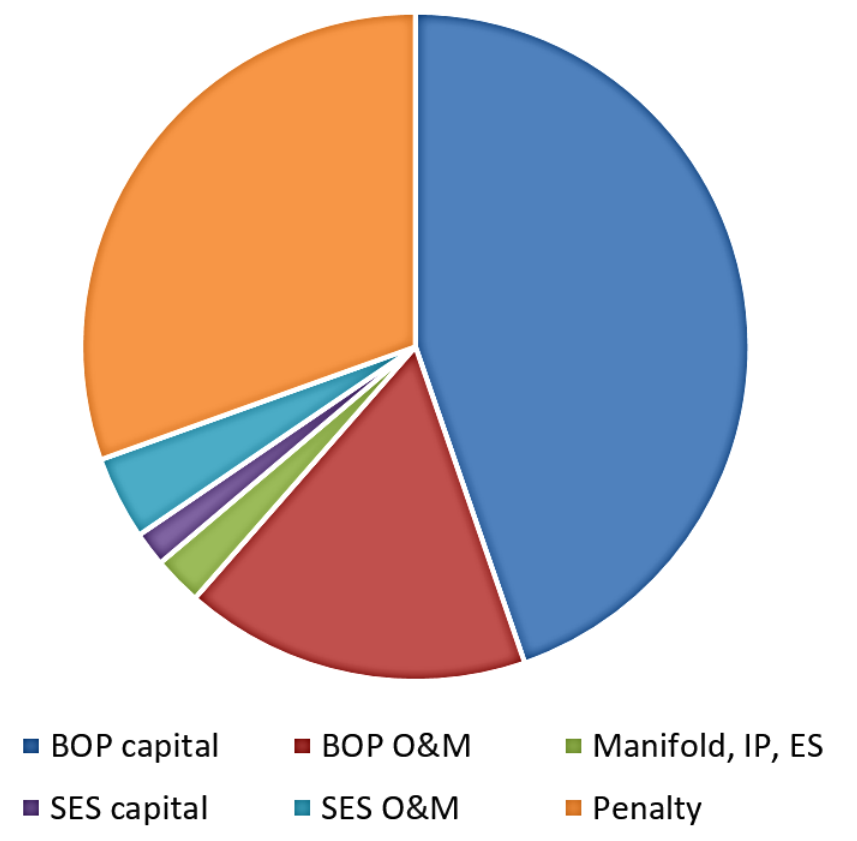

Figure 13 - Fraction of total system costs (discounted) 


\subsection{Scaling demand: $200,000-400,000$}

The preconditioner was also tested for demands of 200,000 and 400,000 while keeping fixed values for the installed capacities (Table 1). The LCOE values for all simulations are reported in Table 3, for both the INL and the ANL preconditioners.

Table 3 - LCOE as a function of the scaling demand (INL vs ANL preconditioner)

\begin{tabular}{ccc}
\hline Scaling demand & INL & ANL \\
\hline 200,000 & 186.29 & $185.25(-0.56 \%)$ \\
\hline 300,000 & 121.29 & $192.14(-36.87 \%)$ \\
\hline 400,000 & 286.76 & $213.86(-25.42 \%)$ \\
\hline
\end{tabular}

For a scaling demand of 200,000, the solution obtained by ANL preconditioner is slightly lower than that of the INL preconditioner $(-0.56 \%)$. The reason for this is that at low demands the unit is always overproducing, and there is not a wide variety of feasible dispatch techniques. In this scenario, at the beginning of the day the overproduced power can be used by both the IP and the ES (Figure 14 and Figure 15). However, during the demand peak, the ANL dispatch prioritizes the energy stored in the battery, which at the beginning of the day was produced by the BOP, over the SES, which has a higher marginal cost (Figure 15 and Figure 16). However, the amount of overproduced power is so high that it does not make a difference whether it is used first by the IP or the ES.

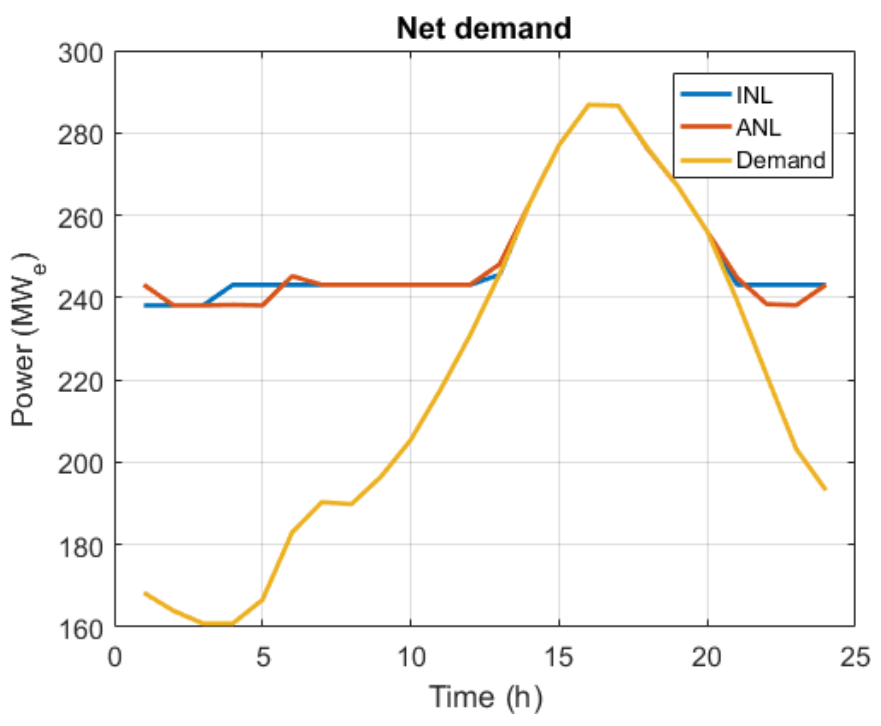

Figure 14 - Total power and net demand as a function of time (200,000 scaling demand) 


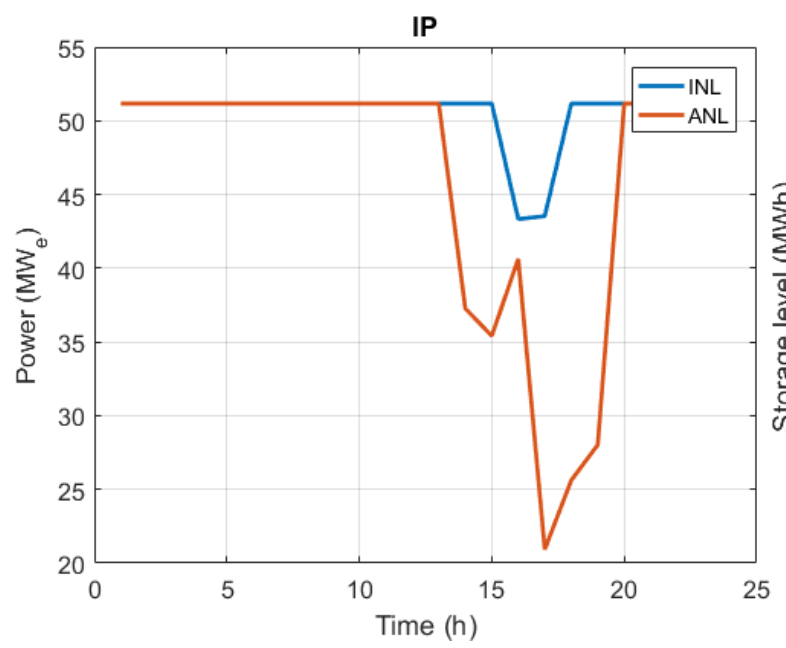

(a)

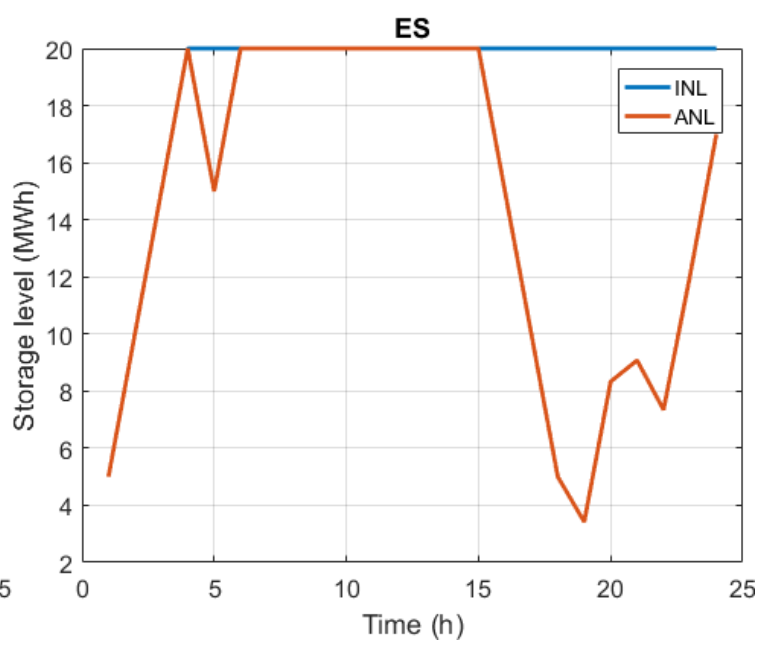

(b)

Figure 15 - IP electric power (a) and ES level (b) as a function of time $(200,000$ scaling demand)

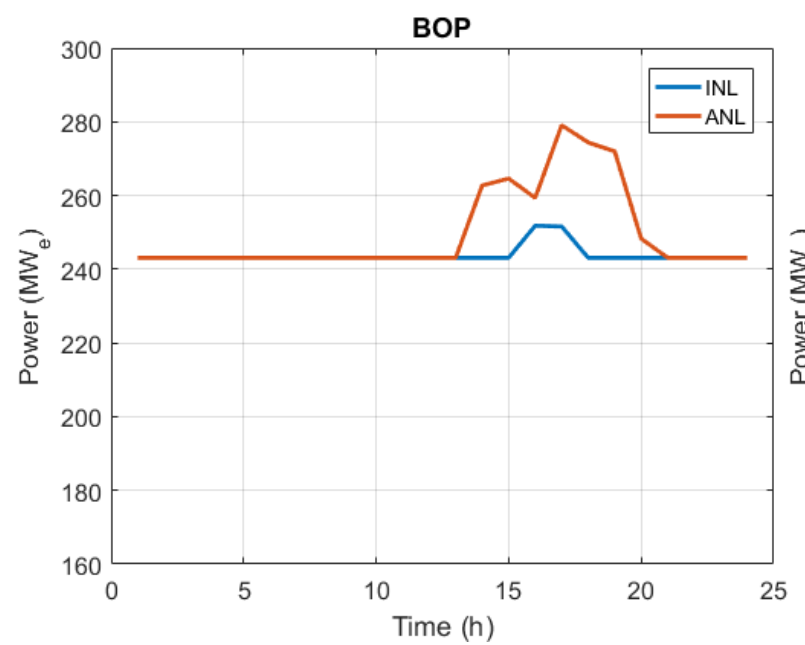

(a)

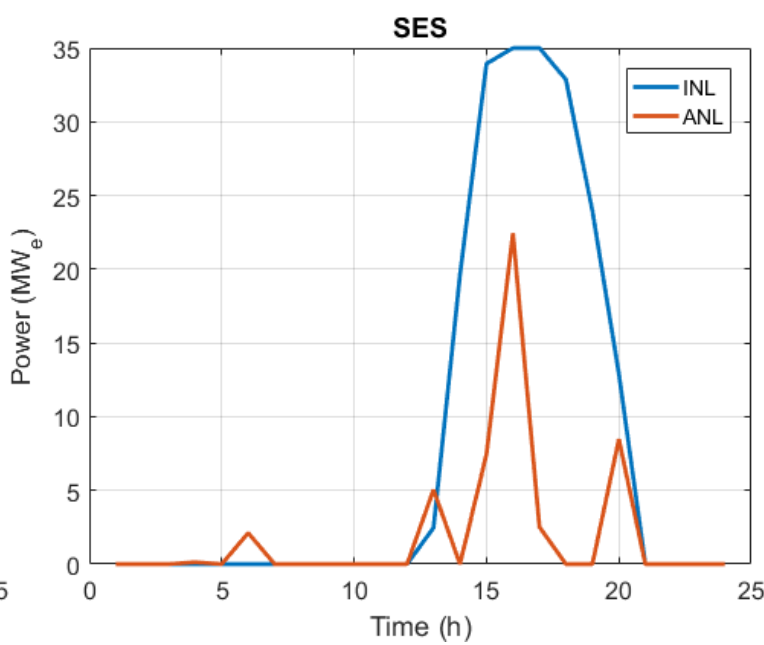

(b)

Figure 16 - BOP (a) and SES (b) dispatched power as a function of time $(200,000$ scaling demand)

Figure 17 through Figure 18 show the results obtained by the ANL and INL preconditioners for a scaling demand of 400,000. Under this demand profile, the system is in a state of underproduction during the whole day. As the previous case, the ANL preconditioner prioritizes the ES over the IP, while the INL one tries to maximize the power of the IP. However, different dispatch decisions are taken during the demand peak that follows. As the demand increase, the dispatch strategy found by the ANL preconditioner allows using the ES over the SES. On the contrary, the INL preconditioner satisfies the demand peak through the SES, which is characterized by high marginal costs. 


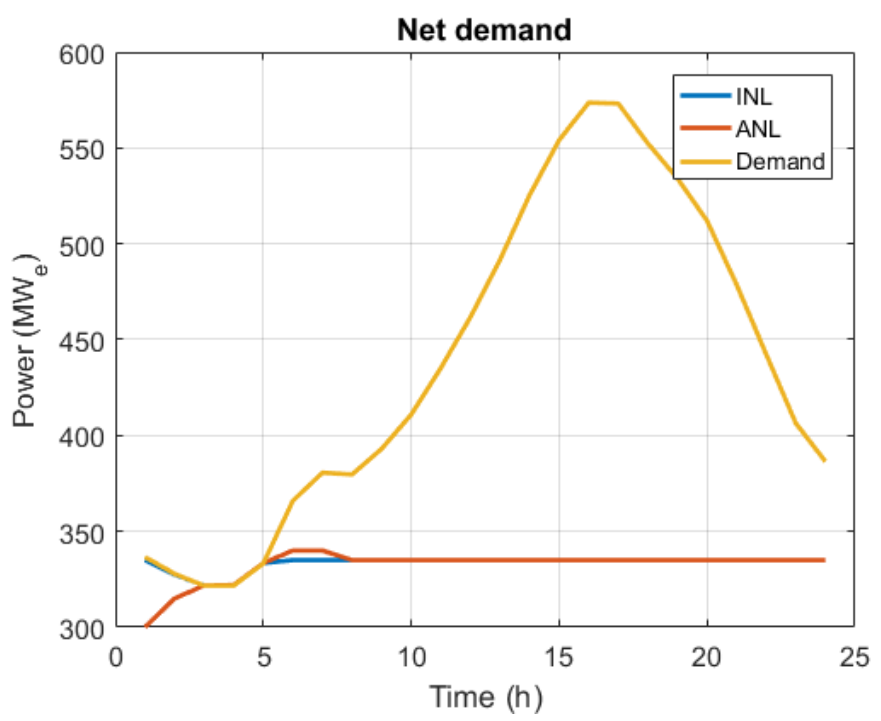

Figure 17 - Total power and net demand as a function of time (400,000 scaling demand)

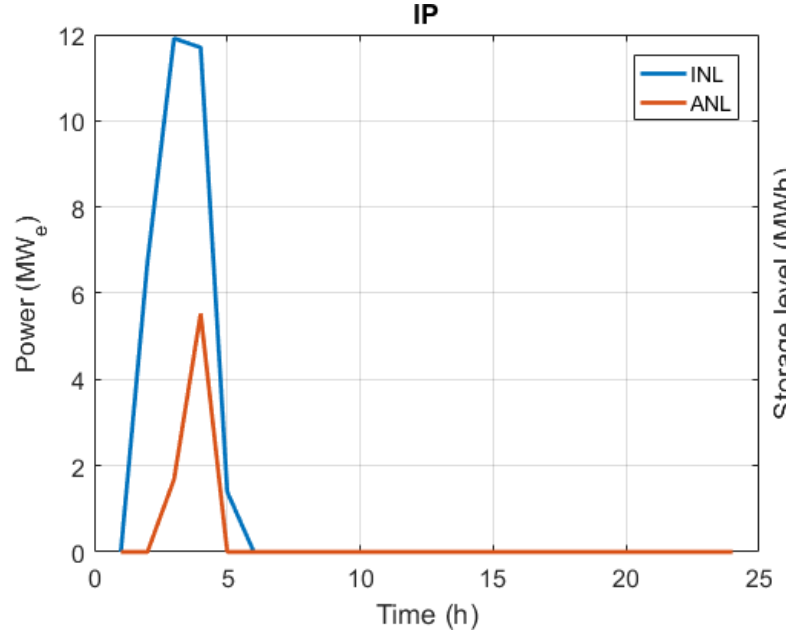

(a)

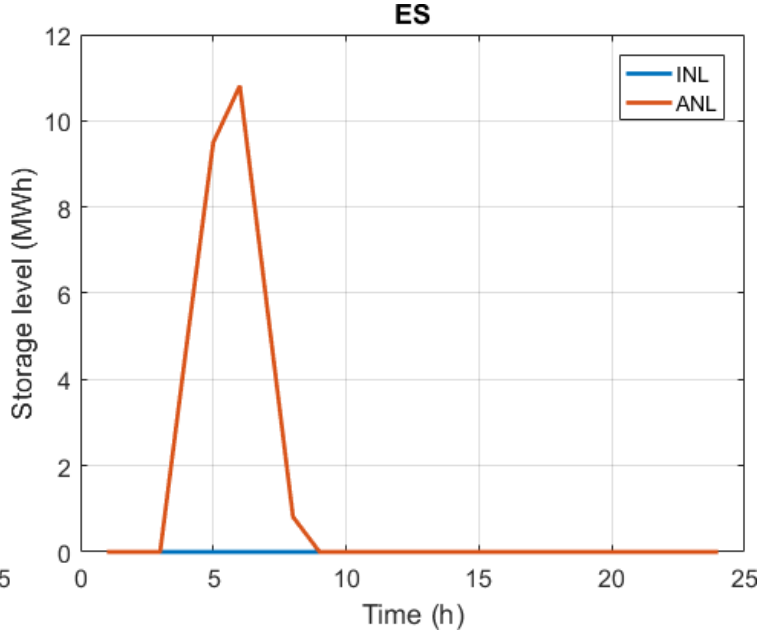

(b)

Figure 18 - IP electric power (a) and ES level (b) as a function of time (400,000 scaling demand) 


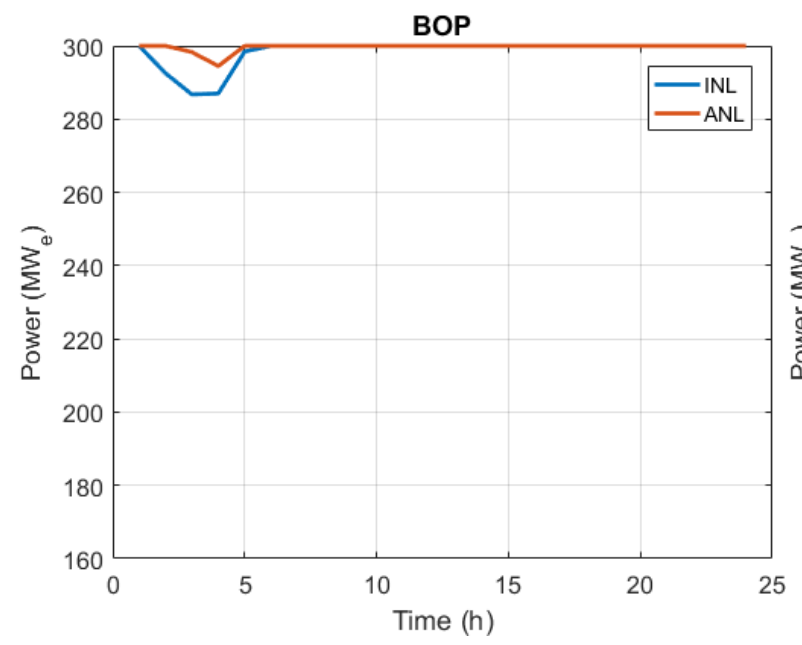

(a)

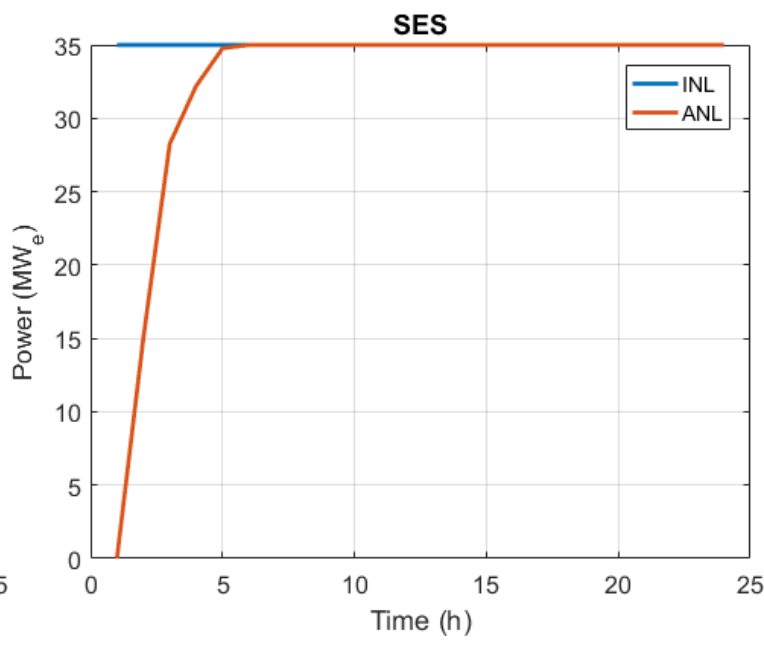

(b)

Figure 19 - BOP (a) and SES (b) dispatched power as a function of time $(400,000$ scaling demand)

\subsection{Computation times}

Using a machine with 8 cores and 188 GB of memory the simulation time was 52,433 seconds for the 300,000 scaling demand case, about 5.2 seconds per iteration. However, the parallel computing functionality of RAVEN was not used at this stage but it will be implemented in the future.

Figure 20 shows the percentage LCOE minimum between the $1^{\text {st }}$ and the $n^{\text {th }}$ iteration as a function of the iteration number, for the three values of scaling demand. For all cases, the LCOE minimum becomes less than $1 \%$ higher than the overall minimum before the $60^{\text {th }}$ simulation. The case with a scaling demand of 400,000 is the slowest to reach the minimum LCOE, which is found at the $4,371^{\text {th }}$ iteration. There is not a defined method to evaluate the minimum number of iteration for convergence. The choice is left to the user's evaluation, depending on the desired level of accuracy of the pre-conditioner output.

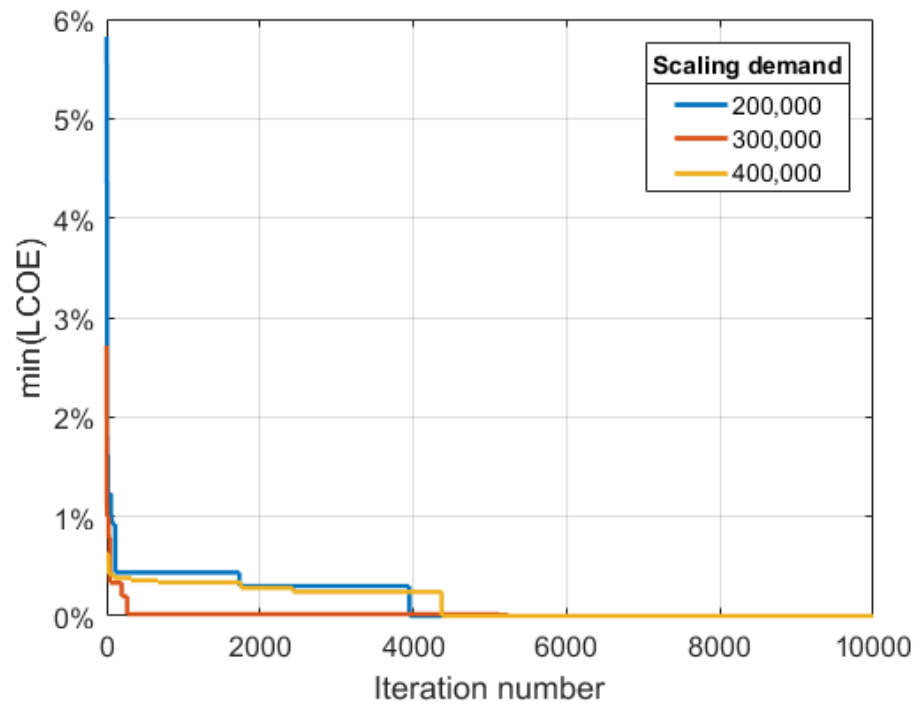

Figure 20 - LCOE minimum as a function of the iteration number 


\subsection{Modified penalty function}

The penalty function was modified to be consistent with the one reported in [8]. A penalty function is expressed by the hyperbolic sine of the absolute value of the missed demand (Eq. (9)).

$$
\operatorname{Penalty}(t)=\sinh \left(\left\|P_{\text {demand }}(M W h)-P_{\text {dispatched }}(M W h)\right\|\right)
$$

This equation provides a more realistic estimate of the price for missed demand. The penalty function expressed by Eq. (2) seems to evaluate the penalty in a 5 minute period, as the argument of the exponent (the missed demand) is divided by 12. Therefore, it seems that the missed demand needs to be expressed in MWh, and the function changes the unit to MW-5 minute. However, the coefficient of the exponent is multiplied by 12 , which suggests that the missed demand needs to be expressed in MW-5 minutes and not in MWh, which is not consistent with the unit of the exponent. A plot of the new penalty function is shown in Figure 21. With respect to the penalty function expressed by Eq. (2), the saturation value is reached for lower values of missed demand.

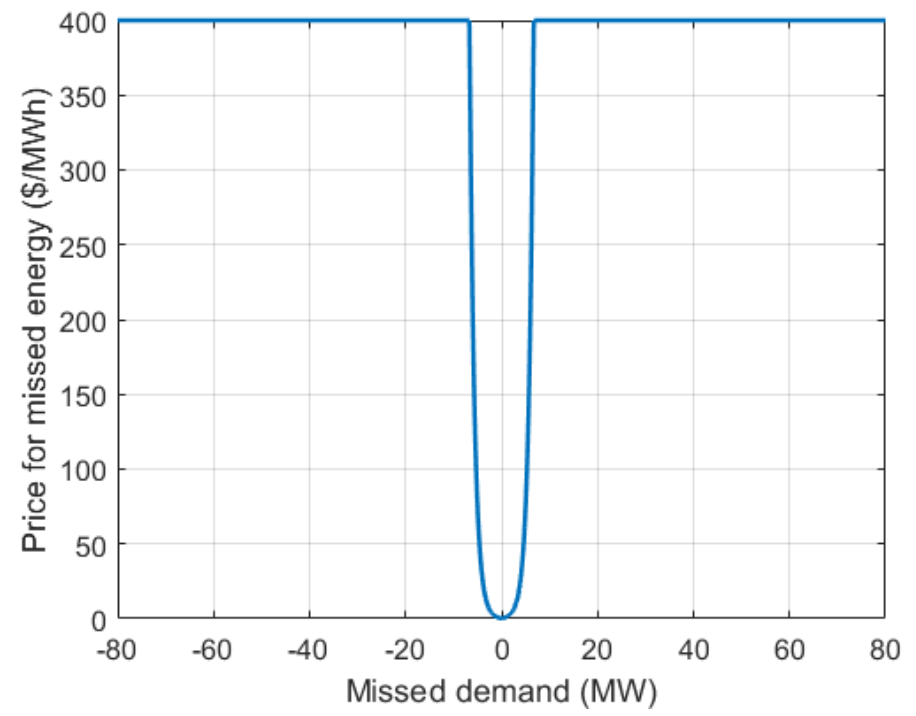

Figure 21 - Modified penalty function for missed demand.

Table 4 - LCOE as a function of the scaling demand (modified penalty function).

\begin{tabular}{ccc}
\hline Scaling demand & INL & ANL \\
\hline 200,000 & 204.60 & $202.37(-1.09 \%)$ \\
\hline 300,000 & 127.83 & $207.06(-38.26 \%)$ \\
\hline 400,000 & 292.30 & $220.00(-32.86 \%)$ \\
\hline
\end{tabular}

The results obtained with the new penalty function are summarized in Table 4. Using the new penalty function, the LCOE is higher for both the ANL and INL cases. Simulating the dispatch with at scaling demand of the 300,000, the INL preconditioner obtains an LCOE of \$207.06/MWh, while that of ANL is $\$ 127.83 / \mathrm{MWh}$, or $38.26 \%$ lower. For the case with a 400,000 scaling 
demand, the main contributor of LCOE is the penalty. As the contribution of the missed demand is higher, the LCOE reduction is lower than the one obtained with the old penalty function. Figure 22 represents the cost fractions for the 300,000 scaling demand case. The increase in LCOE is due to the higher penalty for missed demand, which in this case makes up $29.9 \%$ of the total cost.

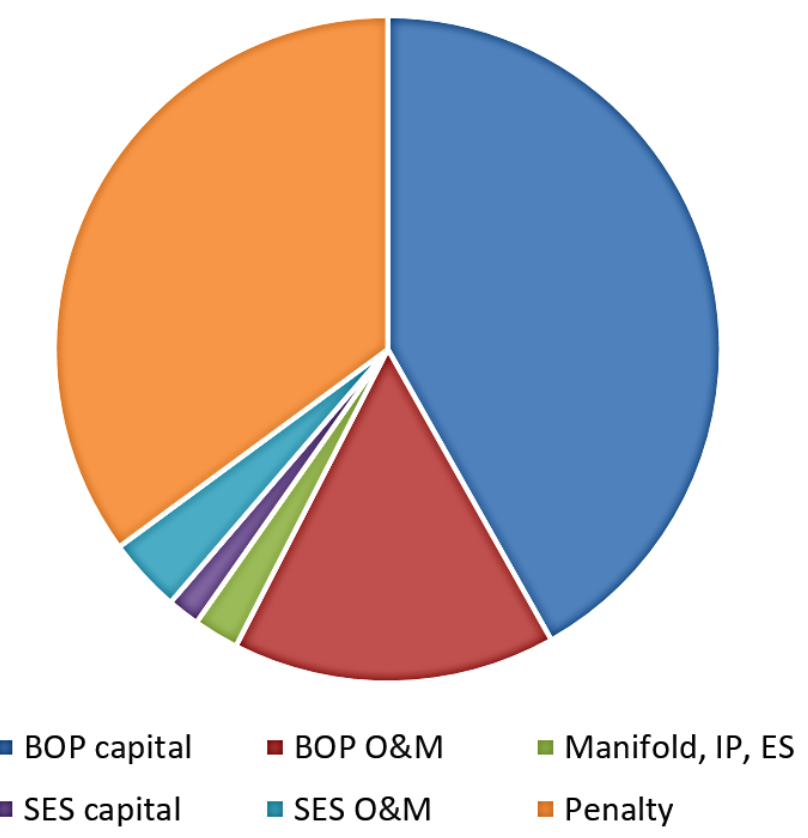

Figure 22 - Fraction of total system costs (discounted) (300,000 scaling demand, modified penalty function). 


\section{Conclusions}

The main goal of the present analysis was the implementation of the ANL preconditioner in the HYBRID framework, to provide an initial solution for the N-R HES power dispatch optimization problem. The algorithm was successfully tested in the HYBRID framework through the analysis of a dispatch problem. The solution of the power dispatch was assessed against the reference solution provided by the INL preconditioner.

The performance of the algorithm based on the results of the test problem are here summarized:

- For every case run, the algorithm provides a better solution to the dispatch problem as compared to the reference approach, i.e. the resulting LCOE is lower;

- The algorithm takes into account the overall net demand profile, present and future, while the reference algorithm considers only the present state of the system. However, the power dispatch was evaluated over a one-day timeframe, which was then extrapolated to the whole lifetime of the system;

- The deterministic approach based on least marginal costs is not valid for all values in the cost input domain. For example, in the test case, because of the low marginal cost of the ES, the deterministic preconditioner does not operate the battery optimally. Furthermore, in case the cost inputs are changed, the algorithm needs to be modified to reflect the change in marginal costs and dispatch logic;

- Because of its stochastic nature, the Monte Carlo-based approach can be used with any value of the inputs variable, i.e. the algorithm does not to be modified as the cost inputs are changed;

- Because of the high impact of missed demand on LCOE, the penalty function for missed demand needs to be properly modeled. In particular, the penalty function used in the HYBRID framework needs to be reviewed.

Future work will extend the dispatch time to a full year and it will include the Dymola modelsimulated system response in the optimization problem. Accounting for the dynamic-imposed constraints will decrease the number of Dymola instances needed in the optimization process, with improvements in computational time. The subsequent decrease in the required time for the iterative optimization process will be evaluated. Future improvements will also address the computational speed of the algorithm in the HYBRID framework by taking advantage of RAVEN parallel computing capabilities. 


\section{Appendix A}

In this appendix, the efforts we spent on the preconditioner implementation into the HYBRID framework are documented. The work is based on the input files used for the analysis in [3]. These input files are accessible in the directory /hybrid/CashFlowCalcs/2017September_INLEXT-1742441/Opt_C_NoModelica_D_TEST of the HYBRID framework.

\section{A1. Preconditioner implementation in HYBRID}

\section{A.1.1. Preconditioner in the RAVEN outer level}

First, a "MC sampler", named 'MCsamp' was added to the input file representing the RAVEN outer level ('HYBRun_noModelica_C_RrR.xml'). The MC sampler is a Monte Carlo sampler that samples the BOP and SES ramp rates according to a uniform distribution between -1 and 1 . The uniform distribution was defined and named 'MC_Distribution'. In the Monte Carlo sampler, the simulation constants were also added. The Monte Carlo based preconditioner Python file '(DispatchANL_MC.py') was saved in the directory.'./../src/' and imported in the simulation. The MultiRun 'OPTrun' was renamed 'MCrun'. The optimizer in the MultiRun was substituted by the Monte Carlo sampler 'MCsamp' previously defined. The preconditioner was then tested. Because of the poor computational performance, it was moved to the inner level of RAVEN.

\section{A.1.2. Preconditioner in the RAVEN INNER level}

In the outer RAVEN input file, the preconditioner was removed. The path of the variables that are passed to the inner level, named "alias variables", was modified. In the original input files, these variables are passed to the inner level input files in the path 'Optimizers|SPSA|constant'. This path was modified to 'Samplers|EnsembleForward|constant'.

The input file representing the inner level of RAVEN is HYBRun_noModelica_D.xml. The 'Ensemble_DYMOLA' EnsembleModel module was renamed 'Dispatch'. The dispatch Python file (DispatchANL_MC.py) is imported in the simulation, and added to the EnsembleModel module, before the DymolaPrep model. The variable group 'GRO_DispatchInit_in_scalar', which represents the input variables of the preconditioner, was added. As previously, the MC sampler was introduced and the uniform probability distribution (between -1 and 1) was defined. The DataObjects representing the preconditioner inputs ('Dispatch_input') and outputs ('Dispatch_output') were defined and the DataObject 'backToMast' was modified to include the power levels of all components ('BOP_XXXX', 'SES_XXXX', 'ES_XXXX', 'IP_XXXX'). Finally, the 'OPTrun' Multirun was renamed 'MCrun'. The input and output DataObjects of the preconditioner were added to the Multirun step. The optimizer step 'opter' was removed from the Multirun, and the Monte Carlo sampler 'MCsamp' was added. 


\section{References}

[1] Epiney, A., Chen, J., Rabiti, C., "Status on the Development of a Modeling and Simulation Framework for the Economic Assessment of Nuclear Hybrid Energy Systems (FY 16)", Idaho National Laboratory, September 2016, INL/EXT-16-39832.

[2] Epiney, A., Rabiti, C., Alfonsi, A., Talbot, P., Ganda, F., (2017), Report on the Economic Optimization of a Demonstration Case for a Static N-R HES Configuration using RAVEN. (INL/EXT-17-41915). Idaho National Laboratory. April 2017.

[3] Rabiti, C., Epiney, A., Talbot, P., Kim, J.S., Bragg-Sitton, S., Alfonsi, A. .. Maronati, G. (2017), Status Report on Modeling and Simulation Capabilities for Nuclear-Renewable Hybrid Energy Systems. (INL/EXT-17-43441). Idaho National Laboratory. September 2017.

[4] Ponciroli, R., Tang, Y., Vilim, R.B. (2018). Characterization of Flexible-Operation Performance of N-R HES Components in Support of a Model-Based Pre-Conditioner. (ANL/NE-18/6). Argonne National Laboratory.

[5] Ponciroli, R., Ganda, F., Vilim, R.B. (2018, September 30-October 4). Development of a Monte Carlo-based economic optimization scheme for nuclear hybrid energy system power dispatch. In American Nuclear Society. Proceedings of the Pacific Basin Nuclear Conference. San Francisco, CA, USA.

[6] Ponciroli, R., Vilim, R.B., Testing of Strategies for the Acceleration of the Cost Optimization. (ANL/NE-17/21). Argonne National Laboratory. August 2017.

[7] Ganda, F., Maronati, G., Economic Data and Modeling Support for the Two Regional Case Studies. (ANL-18/26). Argonne National Laboratory. August 2018.

[8] McJunkin, T., Epiney, A., Rabiti, C. (2017). Report on Integration of Existing Grid Models for N-R HES Interaction Focused on Balancing Authorities for Sub-hour Penalties and Opportunities. (INL/EXT-17-42534). Idaho National Laboratory. June 2017. 


\section{Argonne}

Nuclear Science and Engineering (NSE) Division

Argonne National Laboratory

9700 South Cass Avenue, Bldg. 208

Argonne, IL 60439

www.anl.gov

Argonne National Laboratory is a U.S. Department of Energy laboratory managed by UChicago Argonne, LLC 